\title{
Situación de endeudamiento de las organizaciones de gestión de instalaciones deportivas de ocio no competitivo. Un estudio empírico
}

\section{Situation of the indebtedness of the organizations of sports facilities management of non-competitive leisure. An empirical study}

\author{
Moisés Grimaldi-Puyana ${ }^{1 *}$, Pablo Gálvez-Ruiz², Antonio Jesús Sánchez-Oliver ${ }^{1}$ y Virginia Alcaraz-Rodríguez \\ 1 Departamento de Educación Física y Deporte. Facultad de Educación. Universidad de Sevilla (España). \\ 2 Departamento de Educación en Internet. Facultad de Educación en Internet. Universidad Internacional de La Rioja (España).
}

\begin{abstract}
Resumen: La gestión de la deuda en las organizaciones deportivas es una variable determinante para la supervivencia de éstas, la falta de control puede afectar al buen funcionamiento de una entidad. Según los datos existentes, las organizaciones que gestionan campos de golf gozan de una buena situación económica. Por ello, el propósito del presente estudio fue conocer el estado y evolución de la deuda de las empresas deportivas que participan en el deporte del golf no competitivo. Para ello, se seleccionó una muestra de 93 organizaciones de todo el territorio Espańol, con una antigüedad media de 20,08 años. Para la obtención de los datos se utilizó la información económica del Sistema de Análisis de Balances Ibéricos de la pasada década. Los hallazgos obtenidos en el presente estudio nos llevan a conocer que la deuda media de las organizaciones ha aumentado en el 70,5\% de los casos después de una década. A pesar de la buena salud económica y al aumento de sus ingresos por explotación y total de activos en el período de estudio, podemos afirmar que la deuda de estas organizaciones ha aumentado exponencialmente entre el 2005-2015.
\end{abstract}

Palabras claves: Gestión deportiva, Organizaciones, Endeudamiento, Golf. Abstract: The management of the debt in sports organizations are determinant variables for the survival of these, the lack of control can affect the proper functioning of an entity. According to the existing data, the organizations that manage golf courses enjoy a good economic situation. Therefore, the purpose of this study was to know the status and evolution of the debt of sports companies that participate in the sport of non-competitive golf. For this, a sample of 93 organizations throughout the Spanish territory was selected with an average age of 20,08 years. In order to obtain the data, the economic information of the Iberian Balance Sheet Analysis System of the past decade was used. From the findings obtained in the present study, an increase in the average debt of the organizations of $70,5 \%$ follows after a decade. In spite of the good economic health and the increase of its income by exploitation and total of assets in the period of study, we can affirm that the debt of these organizations has increased exponentially between 2005-2015. Keywords: Sports management, Organizations, Indebtedness, Golf.

\section{Introducción}

La gestión de la deuda, tamaño y estructura en las organizaciones deportivas es una variable determinante para la supervivencia de éstas (Acedo, 2002; Beotas-Lalaguna, BlancoPereira, Cubeiro-Villar, Dorado-Suarez, Gallardo-Guerrero, Lozano-Cid. et al., 2006; IESPORT, 2012; Grimaldi-Puyana y Sánchez-Oliver, 2017a; Ruiz-Huerta y García-Díaz, 2012), dado que una mala gestión por falta de liquidez podría llevar a la desaparición de cualquier organización.

La consecuencia por falta de control podría afectar a la rentabilidad financiera, o lo que es lo mismo, a la capacidad para autofinanciarse o capacidad de innovación e internalización (Huertas y Salas, 2014). Por ello, el control de la deuda y rentabilidad financiara son indicadores fundamentales para accionistas y propietarios de referencia, que ayuda y muestra el rendimiento de una organización empresarial (Amis y Slack, 1996; De La Hoz, Ferrer y De La Hoz-Suárez, 2008; García-Lorenzo, Velar-Martín y Cañadas-Sánchez, 2009;

Dirección para correspondencia [Correspodence address]: Moisés Grimaldi-Puyana. Departamento de Educación Física y Deporte. Facultad de Educación. Universidad de Sevilla (Espańa). mgrimaldi@ us.es
Morillo, 2001; Grimaldi-Puyana y Ferrer-Cano, 2016; Tafunell, 2000; Vithessonthi y Tongurai, 2015).

En general en el sector deportivo, son las grandes organizaciones de gestión de instalaciones deportivas las que presentan índices superiores de endeudamiento según Grimaldi-Puyana y Sánchez-Oliver (2017b); en el mismo sentido López-Prol (2013) señala que la deuda de pequeñas y medianas empresas supone el 3\% del total, situando la mayor parte del endeudamiento en las grandes organizaciones $(57,3 \%$ del total).

Siguiendo con lo publicado en el periódico analizado en junio de 2017, el ranking de organizaciones deportivas más endeudadas con hacienda fueron los clubes deportivos, concretamente los de fútbol y baloncesto: "un total de 19 clubes deportivos aparecen en la lista de grandes morosos publicada por Hacienda. Entre ellos se cuentan 14 clubes de fútbol, cuatro de baloncesto y uno de balonmano. La deuda acumulada de todos ellos con la Agencia Tributaria es de 101,3 millones de euros" (Riaño, 2017).

Por estos motivos, las federaciones deportivas españolas han realizado un esfuerzo con el fin de controlar su deuda (Figura 1), de manera que el balance económico de las propias 
federaciones haya pasado de reflejar 4,5 millones de euros de pérdidas en 2012 a un superávit de 6,2 millones de euros en las cuentas de 2016 (Consejo Superior de Deportes, 2017), lo que quiere decir que la deuda se ha reducido en un 40,08\%, pasando de déficit a superávit (Mundo Deportivo, 2017).

De esta forma, parece que ha llegado el momento que los clubes deportivos a través de una gestión ética y con una actitud proactiva, han reducido su deuda con el fin de mejorar su reputación y sostenibilidad (López-Martínez y Fernández-
Fernández, 2015), todo ello impulsado desde el programa del gobierno espańol para el saneamiento y recapitalización de clubes deportivos reduciendo su deuda (Gay de Liébana, 2012). Desde entonces y a modo de ejemplo, según Maqueda (2017) la deuda con hacienda de los clubes deportivos del fútbol profesional ha descendido un 65\%, pasando de 752 millones de euros en 2011 a 256,8 millones en julio de 2017, datos que fueron reafirmados por Valero (2017) indicando que la deuda de los clubes de fútbol verá su fin en el año 2020.

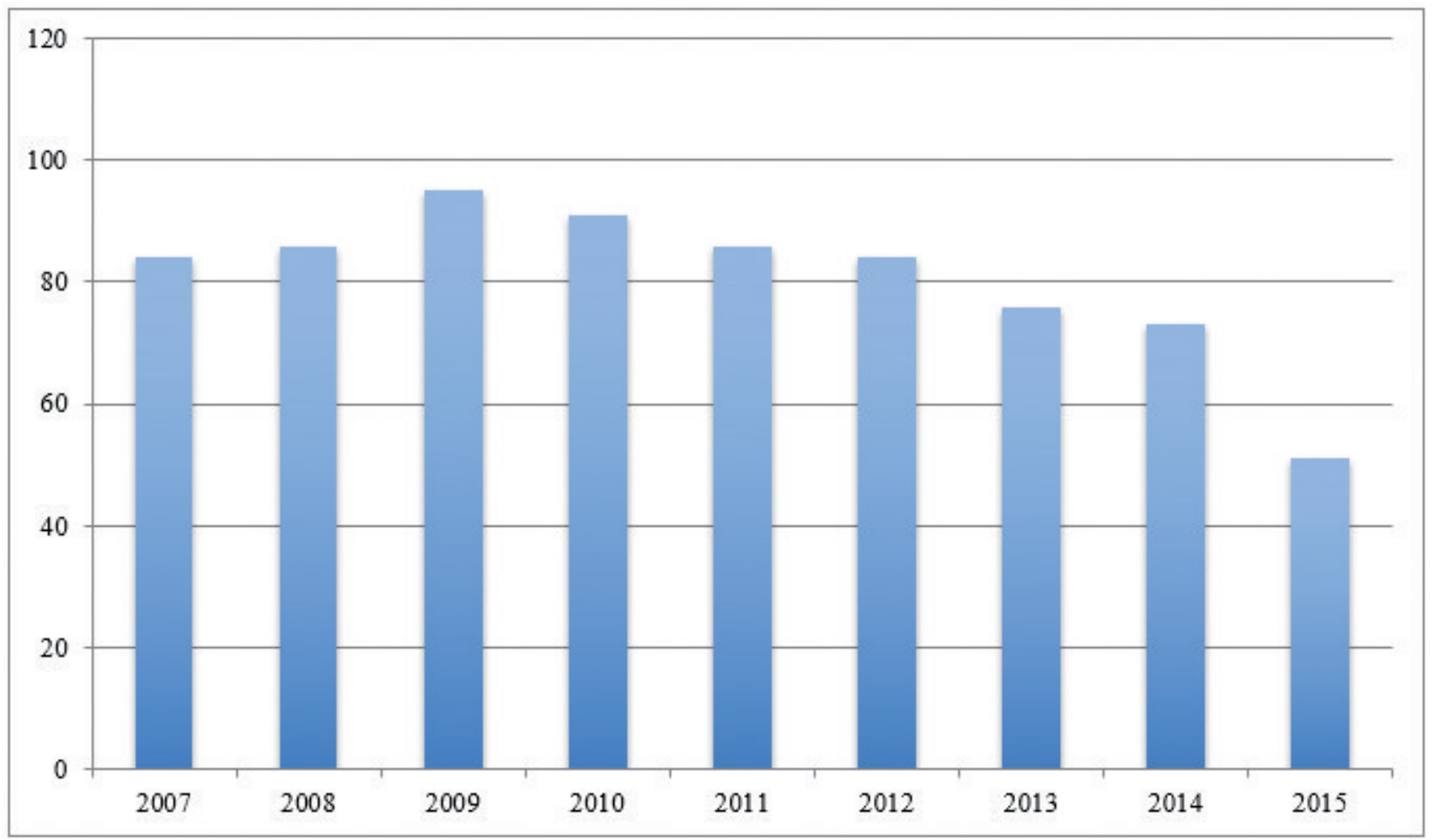

Figura 1. Evolución de la deuda total de las federaciones deportivas españolas en millones de euros (Consejo Superior de Deportes, 2017).

La deuda parece que depende del sector en el que se desarrolle la actividad. En este sentido Segura y Toledo (2003) indican que las empresas de menor tamaño en el sector manufacturero desarrollan su actividad con niveles de deuda superiores a las organizaciones de mayor tamaño. Por otro lado, la deuda también viene determinada por criterios de localización geográfica, como podemos ver que esta más acentuada en Portugal, Italia, Irlanda, Grecia y Espańa. En el caso concreto de Espańa, la deuda pública se situó en un $84 \%$ del Producto Interior Bruto en 2012, siendo ésta el doble que un año antes del comienzo de la crisis económica.

Sin embargo al analizar la industria del golf en el España, se trata de un sector en pleno crecimiento que aporta más de 2.000 millones de euros y sirve de motor a otras industrias (Barciela, 2017), con un aumento del número de campos de golf que completan la oferta de turismo y ocio (Riquel-Ligero y Vargas-Sánchez, 2012), estableciéndose un crecimiento de un 296\% desde 1996 a 2006 (Paniza-Prados, 2005). De ahí, que la Real Federación Española de Golf (2017) cuente hoy en día con más 270.000 jugadores federados, un total de 359 campos de golf, 21 campos rústicos, 95 Pich\&Putt y numerosas escuelas de golf. En el mismo sentido, los autores Grimaldi-Puyana, Sánchez-Oliver, Macías-Plat y Álvarez-García (en prensa) analizaron el perfil, evolución y características de las organizaciones de campos de golf, tras el análisis de las organizaciones espańolas en el período 2005-2015, afirmando que a pesar de la crisis económica y la subida del Impuesto del Valor Añado en 13 puntos (Cordeiro y Asín-Izquierdo, 2016; Europa Press, 2012), éstas no se han visto reducido los valores medios de Ingresos y Activos.

Por lo anteriormente expuesto, y conocida su importancia sobre el control de la deuda en las organizaciones deportivas 
como factor esencial para la supervivencia de las mismas a largo plazo (González-Pérez, Correra-Rodríguez y AcostaMolina, 2002), y a sabiendas de la buena salud en el sector del golf, el propósito del presente estudio fue conocer el estado y evolución de la deuda de las empresas deportivas que participan en el deporte del golf no competitivo.

\section{Metodología}

Para la realización del estudio, la recogida de información se realizó según los Códigos de Actividades Económicas (CNAE) 931 "Actividades deportivas" ( $n=19.178$ ) registrados por el Instituto nacional de Estadística. Se realizó un filtrado selectivo con el fin de obtener todas aquellas que tuviesen relación gestión de instalaciones de golf en España (Figura
2), desde una perspectiva del desarrollo del deporte recreativo no competitivo $(2,13 \%, n=409)$. De esta selección el 52,8\% $(n=216)$ estaban registradas como Gestión de Instalaciones Deportivas (93.11); 7,3\% $(n=30)$ como Actividades de los Clubes Deportivos (931.2), 0,2\% como Actividades de Gestión de los Gimnasios $(n=1)$ y 39,9\% $(n=162)$ a Otras Actividades Deportivas (931.4). Para el análisis de datos se utilizó el programa estadístico SPSS v.21.

Los datos descriptivos de la variable dependiente endeudamiento son presentados en medias y desviación típica para cada uno de los ejercicios económicos disponibles (20052015). Por el contrario, se excluyeron todas aquellas que no tuviesen actividad o no presentaran las cuentas de forma completa en los años 2005 al 2015.

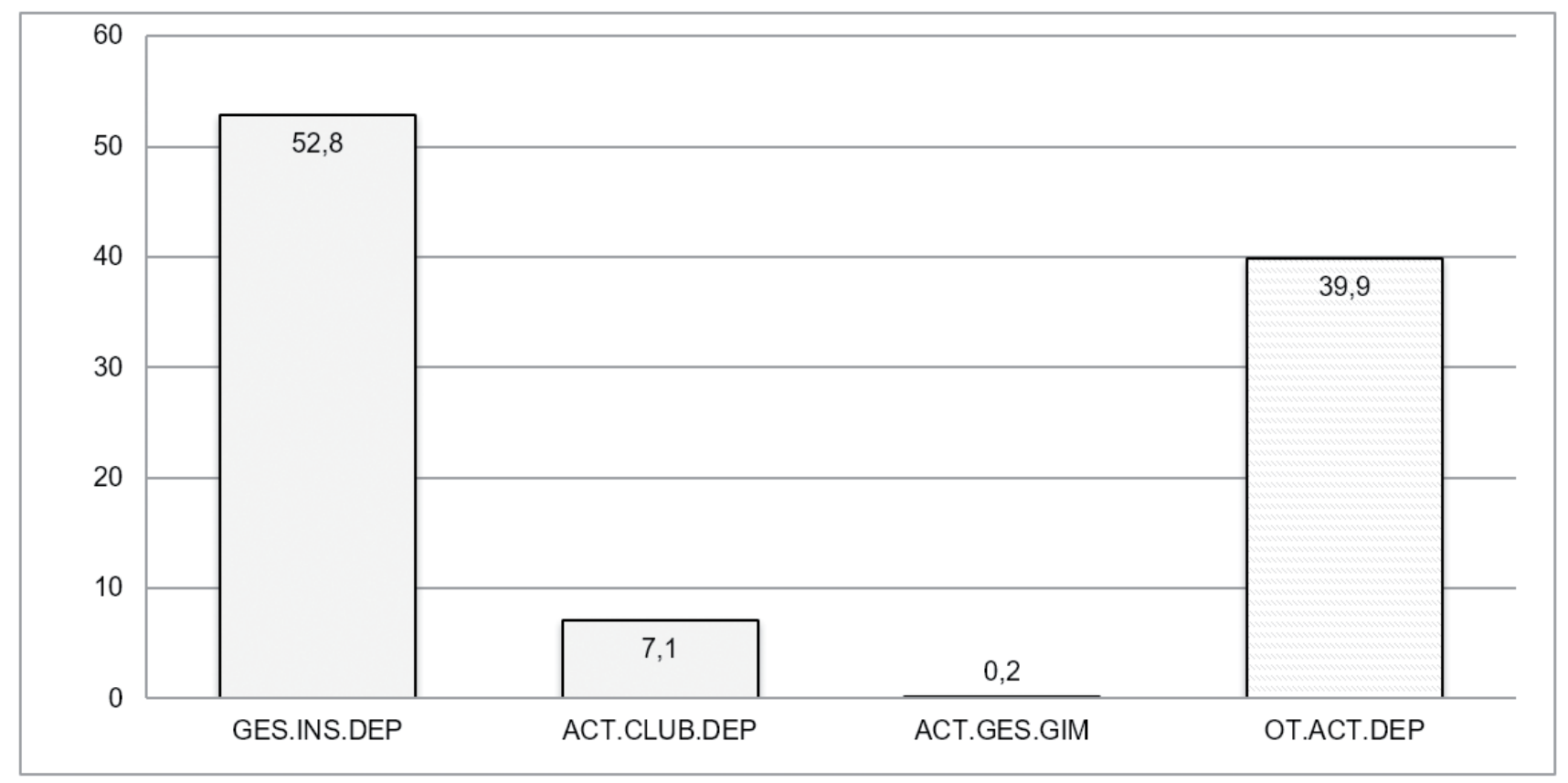

Figura 2. Distribución porcentual de las organizaciones por CNAE

Nota: GES. INS. DEP = Gestión de Instalaciones Deportivas (931.1); ACT. CLUB. DEP = Actividades de los Clubes Deportivos; ACT. GES. GIM = Actividades de Gestión de los Gimnasios; OT. ACT. DEP = Otras Actividades Deportivas.

Una vez determinado el universo objeto de estudio $(n=409)$, se obtuvo la información contable del Sistema de Análisis de Balances Ibéricos (SABI) de la pasada década (2005-2015). El sistema SABI se conoce a nivel internacional como el único producto de estas características y es reconocido desde hace más de 15 ańos como el mejor para análisis financiero. Además se trata de una de las bases de datos más utilizada por la comunidad científica.
Finalmente la muestra seleccionada fue de 93 campos de golf (Tabla 1), con una antigüedad media de 20,08 ańos (DT = 11,1) con la siguiente tipología: 60,2\% fueron campos de 18 hoyos; 10,8\% de 9 hoyos; 9,7\% de Par 3 y Pitch\&Putt; 7,5\% de 27 hoyos; 5,4\% de 72 hoyos y 4,3\% de 27 hoyos. Según la personalidad jurídica, el 50,6\% $(n=47)$ eran Sociedad Limitadas; el 46,2\% $(n=43)$ eran Sociedad Anónima y el 3,2\% Asociaciones $(n=3)$. 
Tabla 1. Relación porcentual y tipología de las organizaciones por Comunidades Autónomas (Grimaldi-Puyana et al., en prensa).

\begin{tabular}{|c|c|c|c|c|c|c|c|c|}
\hline \multirow[t]{2}{*}{ Comunidad Autónoma } & \multirow[t]{2}{*}{ Porcentaje de la Muestra $(n=93)$} & \multicolumn{7}{|c|}{ Relación de la muestra por tipología de campos de Golf } \\
\hline & & $\begin{array}{c}9 \text { hoyos } \\
(\mathrm{n}=10)\end{array}$ & $\begin{array}{c}18 \text { hoyos } \\
(\mathrm{n}=56)\end{array}$ & $\begin{array}{c}27 \text { hoyos } \\
(\mathrm{n}=4)\end{array}$ & $\begin{array}{c}36 \text { hoyos } \\
(\mathrm{n}=7)\end{array}$ & $\begin{array}{c}54 \text { hoyos } \\
(\mathrm{n}=2)\end{array}$ & $\begin{array}{c}72 \text { hoyos } \\
(\mathrm{n}=5)\end{array}$ & $\begin{array}{c}\text { Par P\&3 } \\
(\mathrm{n}=9)\end{array}$ \\
\hline Andalucía & 23,7 & 13,6 & 54,5 & 4,5 & 22,7 & 4,5 & - & - \\
\hline Aragón & 4,3 & 25 & 75 & - & - & - & - & - \\
\hline Asturias & 2,2 & 50 & - & - & - & - & - & 50 \\
\hline Baleares & 10,8 & 90 & - & 10 & - & - & - & - \\
\hline Canarias & 6,5 & - & 100 & - & - & - & - & - \\
\hline Cantabria & - & - & - & - & - & - & - & - \\
\hline Castilla-La Mancha & 3,2 & - & 66,7 & - & - & - & 33,3 & - \\
\hline Castilla y León & 3,2 & - & 33,3 & - & - & - & 66,3 & - \\
\hline Cataluña & 15,1 & 7,1 & 28,6 & & 7,1 & 7,1 & - & 50 \\
\hline Valencia & 7,5 & - & 85,7 & 14,3 & - & - & - & - \\
\hline Extremadura & - & - & - & - & - & - & - & - \\
\hline Galicia & 3,2 & 66,7 & - & 33,3 & & & & \\
\hline La Rioja & - & - & - & - & - & - & - & - \\
\hline Madrid & 12,9 & - & 58,3 & - & - & - & 33,3 & 8,3 \\
\hline Murcia & 2,2 & - & 100 & - & - & - & & \\
\hline Navarra & 2,2 & 50 & 50 & - & - & - & - & - \\
\hline País Vasco & 3,2 & - & 66,7 & - & 33,3 & - & - & - \\
\hline Total & 100 & & & & & & & \\
\hline
\end{tabular}

\section{Resultados}

Los resultados obtenidos ofrecen la evolución del endeudamiento en el periodo comprendido entre 2005 y 2015, como se refleja en la Tabla 2 y en la Figura 3. El endeudamiento medio de organizaciones que gestionan instalaciones deportivas de golf no competitivo españolas aumentan en un 76,6\%. Al comparar el período estudiado, es en $2005(32,2)$ donde se encuentra la tasa menor de endeudamiento, mientras que la superior se sitúa en 2014 (137,9), lo que significa un aumento en la evolución después de diez años de un 70,5\% más endeudadas que la década pasada.

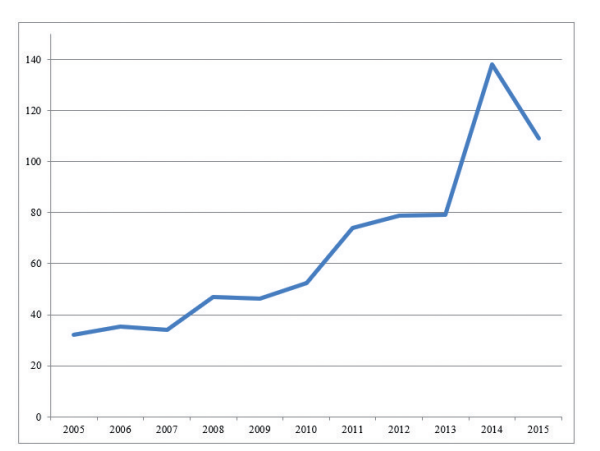

Figura 3. Evolución del endeudamiento en el periodo de 2005-2015 (II).

Nota: Valores expresados en porcentajes $\overline{\mathrm{x}}$ : medias; DT: desviación típica.
Tabla 2. Evolución del endeudamiento en el periodo de 2005-2015 (I).

\begin{tabular}{ccc}
\hline Ańo & $\overline{\mathrm{x}}$ & $\mathrm{DT}$ \\
\hline 2015 & 109,1 & 485,9 \\
2014 & 137,9 & 712,3 \\
2013 & 79,1 & 129,4 \\
2012 & 78,9 & 176,9 \\
2011 & 74,1 & 187,8 \\
2010 & 52,3 & 72,6 \\
2009 & 46,3 & 52,6 \\
2008 & 46,9 & 91,2 \\
2007 & 34,1 & 40,8 \\
2006 & 35,2 & 37,3 \\
2005 & 32,2 & 34,9 \\
\hline
\end{tabular}

Nota: Valores expresados en porcentajes: $\bar{x}$ medias; DT: desviación típica.

Como se observa en la Tabla 3, está representada la evolución de la tasa de endeudamiento medio de organizaciones que gestionan instalaciones deportivas de golf no competitivo españolas segmentadas por tamaño en el período comprendido entre 2005 a 2015. Al analizar por tamaños, son las pequeńas organizaciones las que mayor crecimiento en la tasa media de endeudamiento muestran, siendo 
del 76,6\% al comparar los datos de 2005 respecto a 2015 , y es el año 2014 el de mayor tasa media de endeudamiento $(\bar{x}=171,9)$.

En segundo lugar, son las microempresas las que presentan su tasa media de endeudamiento en un 58,8\% al comparar los datos de 2005 respecto a 2015 y registrando la tasa media mayor en el año $2012(\bar{x}=130,9)$. Sin embargo, las organizaciones medianas y grandes presentan diferente tendencia, en las medianas $(\overline{\mathrm{x}}=-9,6)$ y grandes $(\overline{\mathrm{x}}=-31,1)$ estas reducen su deuda comparando 2005 respecto a 2015, y se presenta su tasa mayor las medianas en $2013(\bar{x}=38,6)$, mientras que para las grandes su tasa mayor se observa en $2011(\overline{\mathrm{x}}=79,5)$.

Tabla 3. Evolución de Endeudamiento segmentadas por tamaño.

\begin{tabular}{|c|c|c|c|c|c|c|c|c|}
\hline \multirow{2}{*}{$\frac{\text { Tamaño }}{\text { Año }}$} & \multicolumn{2}{|c|}{ Micro } & \multicolumn{2}{|c|}{ Pequeña } & \multicolumn{2}{|c|}{ Mediana } & \multicolumn{2}{|c|}{ Grande } \\
\hline & $\overline{\mathrm{x}}$ & $D T$ & $\overline{\mathrm{x}}$ & $D T$ & $\overline{\mathrm{x}}$ & $D T$ & $\overline{\mathrm{x}}$ & DT \\
\hline 2015 & 94,8 & 125,1 & 125,9 & 595,8 & 27,2 & 21,2 & 47,9 & 64,1 \\
\hline 2014 & 89,8 & 108,6 & 171,9 & 877,3 & 25,5 & 21,8 & 45,3 & 61,6 \\
\hline 2013 & 92,5 & 123,6 & 78,6 & 225,3 & 38,6 & 36,1 & 75,6 & 12,2 \\
\hline 2012 & 130,9 & 253,4 & 64,4 & 151,2 & 34,8 & 32,9 & 76,2 & 7,4 \\
\hline 2011 & 117,1 & 295,7 & 62,2 & 144,4 & 34,7 & 35,2 & 79,4 & 9,4 \\
\hline 2010 & 77,7 & 118,1 & 44,5 & 49,8 & 30,3 & 33,4 & 75,5 & 5,5 \\
\hline 2009 & 54,7 & 66,9 & 44,4 & 49,1 & 28,7 & 33,9 & 71,1 & 3,8 \\
\hline 2008 & 80,5 & 170,4 & 36,9 & 37,1 & 27,7 & 32,4 & 33,5 & 47,3 \\
\hline 2007 & 36,8 & 48,6 & 32,1 & 38,6 & 30,1 & 37,9 & 71,9 & 8,4 \\
\hline 2006 & 38,5 & 44,6 & 33,2 & 35,5 & 33,5 & 32,1 & 67,2 & 10,2 \\
\hline 2005 & 38,1 & 40,8 & 29,3 & 33,5 & 29,8 & 29,3 & 62,8 & 3,2 \\
\hline
\end{tabular}

Nota: Valores expresados en porcentajes $\overline{\mathrm{x}}$ : medias; DT: desviación típica.

\section{Discusión}

Tras el análisis de la presente investigación, se puede afirmar que el factor tamaño de las organizaciones deportivas es determinante en la deuda de éstas, y concretamente de las organizaciones que gestionan campos de golf. Al analizar el tamańo, son las microempresas y pequeñas organizaciones las que presentan mayores valores medios de endeudamiento, mientras que las grandes y medianas organizaciones presentan valores medios de endeudamiento respecto a las microempresas.

Estas afirmaciones coinciden con las señaladas por Hamilton y Fox (1998), los cuales concluyen que el tamańo pequeño es un factor que incide en el aumento de la deuda, o Segura y Toledo (2003), en su estudio en otro sector manufacturero, donde concluyen indicando que las empresas más pequeńas son las que tienen mayor deuda y con un coste mayor.

Sin embargo son contrarios a otros ámbitos del sector deportivo, los cuales muestran que las grandes organizaciones de gestión deportiva son las que presentan índices superiores (Grimaldi-Puyana, y Sánchez-Oliver, 2017b), afirmación que sigue la misma línea de López-Prol (2013), que afirma que la deuda de las PYMES es de un 3\% del sector, mientras que las grandes organizaciones acumulan un $57,3 \%$ del sector de la gestión deportiva.

\section{Conclusiones}

Revisada la literatura científica actual, podemos constatar que no existen estudios empíricos que muestren la evolución de la deuda de las organizaciones de golf no competitivo en Espańa. A pesar de la buena salud económica y al aumento de sus ingresos por explotación y total de activos en el período de estudio, podemos afirmar que la deuda de estas organizaciones ha aumentado exponencialmente entre el 2005-2015.

Del mismo modo cabe destacar que el presente trabajo presenta limitaciones, dado que no se puede afirmar que el tamaño de la muestra sea representativo para determinar que se trata de una muestra representativa con un nivel de confianza del 95\% según lo determinado en tamaños muestrales en poblaciones finitas. Además, en la implicación para la gestión este tipo de trabajo podría servir como indicador de gestión económica que ayude a realizar comparaciones entre organizaciones deportivas entre regiones u otros países, con el fin de conocer y diseńar organizaciones con mayor solidez.

Finalmente, el presente trabajo pretende conocer la evolución de la deuda de aquellas organizaciones que gestionan 
campos de golf en España dentro del sector en organizaciones deportivas, así como su comportamiento ante futuros entornos adversos. Estos resultados puede servir como inicio de un indicador fundamenta para la gestión económica, además puede ayudar para el análisis de comparaciones entre organizaciones deportivas de otras regiones o países.

\section{Referencias.}

1. Acedo, M. A. (2002). Rentabilidades, endeudamiento y coste de la deuda de las empresas Riojanas. Análisis empírico por tamaños. En L. Batsch y F. Cossío (Eds.), The Limits of the Firm (pp. 105-113). París: AEDEM Université Paris Dauphine.

2. Amis, J. y Slack, T. (1996). The size-structure relationship in voluntary sport organizations. Journal of Sport Management, 10, 76-86.

3. Barciela, F. (30 de noviembre de 2017). La industria del golf sale del hoyo. La crisis y las nuevas regulaciones para el sector como las relativas al consumo de agua han propiciado la creación de empresas de servicios. El País. Recuperado de https://elpais.com/economia/2017/11/30/actualidad/1512058937_849515.html

4. Beotas-Lalaguna, E, Blanco-Pereira, E, Cubeiro-Villar, J., Dorado-Suarez, A, Gallardo-Guerrero. L., Lozano-Cid., J. et al. (2006). Futuras claves en la gestión de organizaciones deportivas. Espańa: Universidad de Castila-La Mancha.

5. Consejo Superior de Deportes (2016). Las grandes cifras económicas de las federaciones deportivas españolas 2007-2015. Madrid: Ministerio de Educación Cultura y Deporte.

6. Consejo Superior de Deportes (31 de marzo de 2017). El balance económico de las Federaciones Deportivas Españolas pasa de reflejar 4,5 millones de euros en pérdidas en 2012 a un superávit de 6,2 millones en las cuentas de 2016. Recuperado de http://www.csd.gob.es/csd/ documentacion/01GabPr/Novedades/el-balance-economico-de-lasfederaciones-deportivas-espanolas-pasa-de-reflejar-4-8-millones-deeuros-en-perdidas-en-2012-a-un-superavit-de-6-6-millones-en-lascuentas-de-2016/view

7. Cordeiro C. y Asín-Izquierdo, I. (21 de abril del 2016). De la subida del IVA a la evolución del sector: Una aproximación a los últimos años. [Mensaje de un blog]. Recuperado de http://gymfactory.net/revista/ gesti\%C3\%B3n/75-evoluci\%C3\%B3n/383-de-la-subida-del-iva-ala-evoluci $\% \mathrm{C} 3 \% \mathrm{~B} 3 \mathrm{n}$-del-sector-una-aproximaci\%C3\%B3n-a-los$\%$ C3\%BAltimos-a\%C3\%B1os

8. De La Hoz, B., Ferrer, M. A. y De La Hoz-Suárez, A. (2008). Indicadores de rentabilidad: herramientas para la toma decisiones financieras en hoteles. Revista de Ciencias Sociales (RCS), 14(1), 88-109.

9. Europa Press (25 de julio de 2012). La Federación Nacional de Empresarios de Instalaciones Deportivas muestra su desacuerdo con la subida del IVA. La voz libre Europa press. Recuperado de http://www. lavozlibre.com/noticias/ampliar/619567/la-federacion-nacional-deempresarios-de-instalaciones-deportivas-muestra-su-desacuerdo-conla-subida-del-iva

10. García-Lorenzo, R, Velar-Martín, L. N. y Cañadas-Sánchez, A.F. (2009). Análisis de los estados contables en el nuevo PGC 2008. Madrid: ESIC Editorial.

11. Gay de Liébana, J. (2012). España se escribe con E de endeudamiento. Barcelona: Deusto.

12. González-Pérez, M., Correra-Rodríguez, A. y Acosta-Molina, A.L. (2002). Factores determinantes de la rentabilidad financiera de las Pymes, Resvista Española de Financiación y Economía, 31(112), 395429.

13. Grimaldi-Puyana, M y Sánchez-Oliver, A. J. (2017b). Evolución en rentabilidad financiera y endeudamiento según tamaño de organizaciones de gestión de instalaciones deportivas de ocio no competitivo. PODIUM Sport, Leisure and Tourism Review, 6(3), 30-45.

14. Grimaldi-Puyana, M. y Ferrer-Cano, P. (2016). Dimensión y Perfil de las Organizaciones de Gestión de Instalaciones y Deportiva. Apunt, Educación Física y deporte, 4(126), 72-78.

15. Grimaldi-Puyana, M. y Sánchez-Oliver, A. (2017a). Tamaño y evolución económica de los clubes de baloncesto S.A.D. después de la crisis económica de 2008. Cuadernos de Psicología del Deporte, 17(3), 137-142.

16. Grimaldi-Puyana, M., Sánchez-Oliver, J. A., Macías-Plat, R., y Álvarez-García, J. (en prensa). Perfil, evolución y características de las organizaciones de gestión de campos del golf en España. Revista Espacios.

17. Hamilton, R. T. y Fox, M. A. (1998). The Financing Preferences of Small Firm Owners. International Journal of Entrepreneurial Behavior \& Research, 4(3), 239-248.

18. Huerta, P., Contreras, S. Almodóvar, J. y Navas, J. (2010). Influencia del tamańo empresarial sobre los resultados: un estudio comparativo entre empresas chilenas y españolas. Revista Venezolana de Gerencia, 15(50), 207-230.

19. Huertas, E. y Salas, D. (2014). La legitimidad de empresas y empresarios en Espańa: una perspectiva comparada. Centro Cívico Opinión, 14, 17-47.

20. IESPORT (17 de enero de 2012). Gestión de la deuda. [Mensaje en un blog]. Recuperado de http://www.iesport.es/metodologia/188.html

21. Límites y problemas en el contexto de la crisis económica. Revista de Estudios Autonómicos y Federales, 15, 124-163.

22. López-Martínez y Fernández-Fernández J. L. (2015). Responsabilidad Social Corporativa y buen gobierno de los clubes de futbol españoles. Universia Business Review, 2, 38-53.

23. López-Prol, J. (2013). Crisis de deuda pública del estado español y la Unión Europea. ¿Causa o consecuencia? Papeles de Europa, 26(2), 93102.

24. Maqueda, A. (9 de octubre de 2017). La deuda de los clubes de fútbol con Hacienda baja a 257 millones de euros. El País. Recuperado de https:// elpais.com/economia/2017/10/09/actualidad/1507573314_096613. html

25. MECD (2016). Anuario de Estadisticas deportivas. Madrid: Ministerio de Educación, Cultura y Deporte. Disponible en: https://www.mecd. gob.es/servicios-al-ciudadano-mecd/estadisticas/deporte/anuario-deporte.html

26. Morillo, M. (2001). Rentabilidad Financiera y Reducción de Costos. Actualidad Contable Faces, 4(4), 35-48.

27. Mundo Deportivo (3 de marzo de 2017). Las federaciones españolas, del déficit al superávit en cuatro años. El mundo deportivo. Recuperado de http://www.mundodeportivo.com/mas-deporte/20170331/421337022701/las-federaciones-espanolas-del-deficit-alsuperavit-en-cuatro-anos.html.

28. Paniza-Prados, J. L. (2005). La percepción social del golf en Andalucía. Sevilla: Consejería de Turismo, Comercio y Deporte.

29. Real Federación Española de Golf (2017). Evolución de licencias. Recuperado de: http://www.rfegolf.es/ArtculosDocumento/LICENCIAS/ evolucionlicencias.pdf.

30. Riaño, M. (23 de Julio de 2017). Los equipos de fútbol y baloncesto que más dinero deben a Hacienda. Recuprrado de: https://www.elindependiente.com/economia/2017/06/23/los-clubes-deportivos-deben-hacienda-1013-millones-euros/

31. Riquel-Ligero, A. y Vargas-Sánchez, A. (2012). The institutional framework of environmental character on andalusian golf courses: a factor analysis, Cuadernos de Turismo, 29, 287-289. 
32. Ruiz-Huerta, J. y García-Díaz, M. (2012). El endeudamiento de las Comunidades Autónomas: el endeudamiento de las comunidades autónomas: límites y problemas en el contexto de la crisis económica, REAF, 15(4), 124-163.

33. Segura, J. y Toledo, L. (2003). Tamaño, estructura y coste de financiación de las empresas manufactureras espańola. Investigaciones Económicas, 27(1), 39-69.

34. Tafunell, X. (2000). La rentabilidad financiera de la empresa española, 1880-1981: Una estimación en perspectiva sectorial. Revista de Historia Industrial, 18, 71-112.
35. Valero, A. (2017). Clemente Villaverde, sobre Hacienda: En 2020 estará finiquitada la deuda de los clubes. El Mundo. Recuperado de: http:// www.elmundo.es/deportes/futbol/2017/07/12/59664e0eca4741ed0d8 b45ee.html

36. Vithessonthi, C. y Tongurai, J. (2015). The effect of firm on the leverage-performance relationship during the financial crisis of 2007-2009. Journal of Multinational Financial Management, 29, 1-29. 